\title{
Fast GPU-beamforming of $\backslash$ Row-Column Addressed Probe Data
}

Stuart, Matthias Bo; Jensen, Patrick Møller; Olsen, Julian Thomas Reckeweg ; Kristensen, Alexander Borch; Schou, Mikkel; Dammann, Bernd; Sørensen, Hans Henrik Brandenborg; Jensen, Jørgen Arendt

\section{Published in:}

Proceedings of 2019 IEEE International Ultrasonics Symposium

Link to article, DOI:

10.1109/ultsym.2019.8925802

Publication date:

2019

Document Version

Peer reviewed version

Link back to DTU Orbit

Citation $(A P A)$ :

Stuart, M. B., Jensen, P. M., Olsen, J. T. R., Kristensen, A. B., Schou, M., Dammann, B., Sørensen, H. H. B., \& Jensen, J. A. (2019). Fast GPU-beamforming of $\backslash$ Row-Column Addressed Probe Data. In Proceedings of 2019 IEEE International Ultrasonics Symposium (pp. 1497-1500). IEEE. https://doi.org/10.1109/ultsym.2019.8925802

\section{General rights}

Copyright and moral rights for the publications made accessible in the public portal are retained by the authors and/or other copyright owners and it is a condition of accessing publications that users recognise and abide by the legal requirements associated with these rights.

- Users may download and print one copy of any publication from the public portal for the purpose of private study or research.

- You may not further distribute the material or use it for any profit-making activity or commercial gain

- You may freely distribute the URL identifying the publication in the public portal 


\title{
Fast GPU-beamforming of Row-Column Addressed Probe Data
}

\author{
Matthias Bo Stuart*, Patrick Møller Jensen ${ }^{\dagger}$, Julian Thomas Reckeweg Olsen ${ }^{\dagger}$, Alexander Borch Kristensen*, \\ Mikkel Schou*, Bernd Dammann ${ }^{\dagger \ddagger}$, Hans Henrik Brandenborg Sørensen ${ }^{\ddagger}$, and Jørgen Arendt Jensen* \\ ${ }^{*}$ Center for Fast Ultrasound Imaging, DTU Health Technology, Technical University of Denmark \\ ${ }^{\dagger}$ DTU Compute, Technical University of Denmark \\ ${ }_{\ddagger}^{\ddagger}$ DTU Computing Center, Technical University of Denmark
}

\begin{abstract}
A delay-and-sum beamformer for 3D imaging using row-column arrays and written in CUDA is presented and compared to an existing similar GPU-based beamformer written in the MATLAB programming language. Data from a 192+192 rowcolumn array single element emission sequence is simulated and beamformed. The two beamformers' performance is evaluated in two synthetic aperture setups comprised of 1) two orthogonal planes and 2) a full volume on three different NVIDIA GPUs: a $1050 \mathrm{Ti}$, a $1080 \mathrm{Ti}$, and a TITAN V. The execution time and the sample throughput (samples beamformed per second) are reported. The CUDA beamformer performs consistently better than the MATLAB beamformer with speed-ups ranging from 1.9 to 64.6 times, and the worst-case throughput of the CUDA beamformer exceeds the best-case of the MATLAB beamformer. High-resolution images of crossing planes can be beamformed at up to $13 \mathrm{~Hz}$, while a 50-by-50-by-20 cubic-millimeter highresolution volume sampled at one quarter of a millimeter is beamformed in 3 seconds.
\end{abstract}

\section{INTRODUCTION}

$3 \mathrm{D}$ ultrasound imaging requires a $2 \mathrm{D}$ distribution of transducer elements to steer the beam in both azimuth and elevation. Fully populated arrays can be full matrices [1] or crossed electrodes that are also known as row-column (RC) arrays [2]. While the interconnect scales quadratically with the number of transducer elements for matrix arrays, it scales linearly for $\mathrm{RC}$ arrays. The number of elements translates to resolution, contrast, and better signal-to-noise ratio and thus penetration, where a RC array performs better than a matrix array with the same number of elements [3]. Flat RC arrays are limited to a forward-facing field-of-view, which can be remedied by applying a defocusing lens [4], [5]. The imaging performance of RC arrays has been verified by a number of groups [6]-[9], and volumetric $3 \mathrm{D}$ vector flow imaging has been demonstrated [10], [11].

Another advantage of RC arrays' linear scaling is that volumetric images are made from an equivalent amount of data to that used for $2 \mathrm{D}$ imaging. This greatly alleviates the issue of the memory wall [12] for 3D ultrasound imaging making frame rates depend primarily on processing speed instead of memory bandwidth. Beamforming is inherently parallel since no computational dependency exists between output samples, and parallel processors such as GPUs can deliver real-time 2D imaging [13], [14]. This work investigates and compares the performance of two RC beamformers on three different GPUs.
Section II presents the two beamformers being investigated, Section III describes the experiments conducted to evaluate their performance and the performance metrics used, Section IV presents the results, Section V discusses the results, and Section VI offers conclusions.

\section{BEAMFORMERS}

Two delay-and-sum (DAS) beamformers are written for execution on GPUs. Both beamformers take as input the geometry (transmit focus, receive element coordinates, and image geometry), apodization parameters, speed of sound, and sampling frequency of the data. Dynamic apodization is supported by both beamformers parametrized on the F-number and window function.

The RC array's long elements result in cylindrically shaped waves - rather than the spherical waves from small elements which requires a different delay model for DAS beamforming [15]. A side effect of this is that the delay calculation can be reduced to a $2 \mathrm{D}$ problem through appropriate choice of the coordinate system [15], [16]. This reduction is used in both beamformers.

One beamformer is written entirely in the MATLAB programming language using the gpuarray type for GPU processing and has been presented elsewhere [16]. The other beamformer is written in C++ using NVIDIA's CUDA extensions and called from MATLAB using the MEX interface. These two beamformers perform the same calculations with some differences in implementation outlined below. They are referred to as the MATLAB and the CUDA beamformer, respectively. An overall description is given of the MATLAB beamformer, while the CUDA beamformer is described in more detail.

\section{A. MATLAB beamformer}

The MATLAB beamformer has been presented and evaluated on a single GPU previously [16] and is briefly summarized here. All intermediate results (transmit, receive, and total delays and apodization values) are stored in memory on the GPU for all output samples to minimize recomputations at the cost of increased memory transfers. This essentially creates memory and apodization tables that are only recalculated, if the related input parameters change. Apodization values are calculated by evaluating the window function with the 
appropriate parameters. Sub-sample precision is attained using cubic interpolation, and the image geometry is specified as a list of the output samples' coordinates.

\section{B. CUDA beamformer}

CUDA uses a single instruction, multiple threads (SIMT) execution model, where all program threads execute the same set of instructions. For efficient execution, threads should have equal (or similar) workload and have a minimum of synchronization among other considerations. The general beamforming problem has two immediate approaches for distribution across threads: 1) for a window of input samples, calculate the output values these input samples contribute to, and 2) for a given output sample, load the corresponding input samples and calculate the output value. The first approach is likely to result in uneven workloads between threads and requires synchronization for summing the different contributions to each output value. The second approach has equal workload for all threads and requires no synchronization. The CUDA beamformer therefore calculates one output sample per thread, i.e., each thread calculates the sum across all receive channels.

The intermediate results (delays and apodization values) are recalculated every time they are needed. The calculation is performed based on a minimal set of parameters (tens of bytes) as opposed to the delay and apodization tables read from memory by the MATLAB beamformer. The image geometry is specified as a set of lines with an origin and a step vector, where all lines must have the same number of samples, and 3rd order Lagrange interpolating polynomials are used for subsample interpolation.

A cumulative sum of images is maintained in GPU memory for synthetic aperture (SA) imaging [17], [18] to avoid transferring each low-resolution image out of the GPU.

\section{EXPERIMENTS}

The two beamformers are evaluated on three different NVIDIA GPUs: a GeForce $1050 \mathrm{Ti}$, a GeForce $1080 \mathrm{Ti}$, and a TITAN V. CUDA toolkit version 9.0 and MATLAB R2018a are used.

A single-element SA imaging sequence with a $192+192$ $\lambda / 2 \mathrm{RC}$ array is used for two use-cases: 1) beamforming two orthogonal planes and 2) beamforming a volume. The output is sampled at $\lambda / 2$ in all directions. In setup 1 , the $\mathrm{XZ}$ and $\mathrm{YZ}$ planes contain $192 \times 76$ samples each. In setup 2 , the volume is $192 \times 192 \times 76$ samples $(x \times y \times z)$. For the MATLAB beamformer, the volume in setup 2 needs to be split in subvolumes due to the memory used for intermediate results. For the 1050 Ti with 4 GB memory, 21 sub-volumes are needed to cover the full volume, the $1080 \mathrm{Ti}$ with 11 GB memory needs 8 sub-volumes, and the TITAN V with 12 GB needs 7 subvolumes. The inputs to the beamformers are analytic signals from a Field II simulation [19], [20] represented using double precision floating point numbers.

For performance measurements, execution times are measured using the wall-clock time, while the respective systems are otherwise unused. The same beamforming operations and
TABLE I

EXECUTION TIMES IN SECONDS FOR SETUP 1

\begin{tabular}{l||ccc|ccc||c}
\multicolumn{1}{l||}{ GPU } & \multicolumn{3}{|c|}{ MATLAB } & \multicolumn{3}{|c|}{ CUDA } & Speed up \\
\hline \hline $1050 \mathrm{Ti}$ & 6.7 & \pm & 0.046 & 2.4 & \pm & 0.003 & 2.8 \\
\hline $1080 \mathrm{Ti}$ & 7.2 & \pm & 0.012 & 0.65 & \pm & 0.001 & 11 \\
\hline TITAN V & 4.7 & \pm & 0.007 & 0.073 & \pm & 0.001 & 65
\end{tabular}

TABLE II

EXECUTION TIMES IN SECONDS FOR SETUP 2

\begin{tabular}{l||ccc|ccc||c}
\multicolumn{1}{l||}{ GPU } & \multicolumn{3}{c|}{ MATLAB } & \multicolumn{3}{c|}{ CUDA } & Speed up \\
\hline \hline $1050 \mathrm{Ti}$ & 411 & \pm & 0.141 & 216 & \pm & 0.009 & 1.9 \\
\hline $1080 \mathrm{Ti}$ & 99.5 & \pm & 0.007 & 42 & \pm & 0.001 & 2.4 \\
\hline TITAN V & 52.9 & \pm & 0.231 & 3.1 & \pm & 0.004 & 17
\end{tabular}

read-out of the results to main memory were repeated 10 times in a loop, and each loop iteration was timed. The first three iterations were discarded to eliminate initialization effects, while the mean and standard deviation were calculated from the remaining seven iterations. Reads and writes from and to disk are not included in the timing, and a routine to flush the CPU caches was called between each iteration to avoid a false speed-up from beamforming the same data repeatedly.

The sample throughput, $N_{s p s}$, (number of samples beamformed per second) is calculated as

$$
N_{s p s}=\frac{N_{l} N_{s} N_{r x} N_{t x}}{\bar{t}},
$$

where $N_{l}$ is the number of image lines, $N_{s}$ is the number of samples per line, $N_{r x}$ is the number of receive elements, $N_{t x}$ is the number of transmit events (the number of lowresolution images used to make a high-resolution image), and $\bar{t}$ is the mean execution time.

Denoting the mean execution time of the MATLAB beamformer $\bar{t}_{M}$ and of the CUDA beamformer $\bar{t}_{C}$, the speed-up, $s$, is calculated as

$$
s=\frac{\bar{t}_{M}}{\bar{t}_{C}} .
$$

\section{RESUlts}

Fig. 1 shows the YZ planes of setup 1 for both beamformers. The point spread functions (PSFs) have only small deviations with a mean difference of $-79 \mathrm{~dB}$ relative to the peak of the envelopes. The two beamformers have slightly different implementations of the calculations as described in Section II, which may be the cause of these deviations. Both PSFs have the same resolution measured as the full-width at halfmaximum (FWHM) of $2.4 \lambda$ in the $y$-direction and $0.66 \lambda$ in the $z$-direction, where $\lambda$ is the wavelength at the transducer's center frequency. The $20 \mathrm{~dB}$ cystic resolution [21] is $1.9 \lambda$ in the YZ plane for both beamformers.

Fig. 2 shows the performance of the beamformers on both setups. The vertical axis shows the sample throughput for each setup and GPU. Tables I and II show the mean and standard deviation of the execution time for each GPU for setups 1 and 2 , respectively.

The standard deviations on the execution times are very low, showing consistent running times, which is to be expected of 

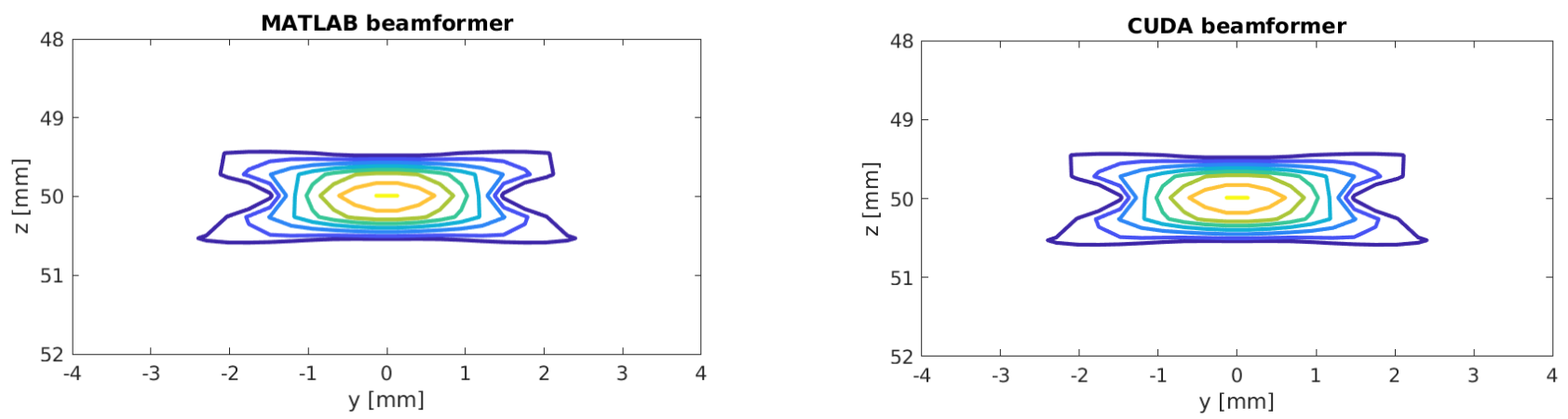

Fig. 1. PSF from the MATLAB (left) and CUDA (right) beamformers for the YZ plane of setup 1. Contour lines are shown at $6 \mathrm{~dB}$ intervals down to $-42 \mathrm{~dB}$.

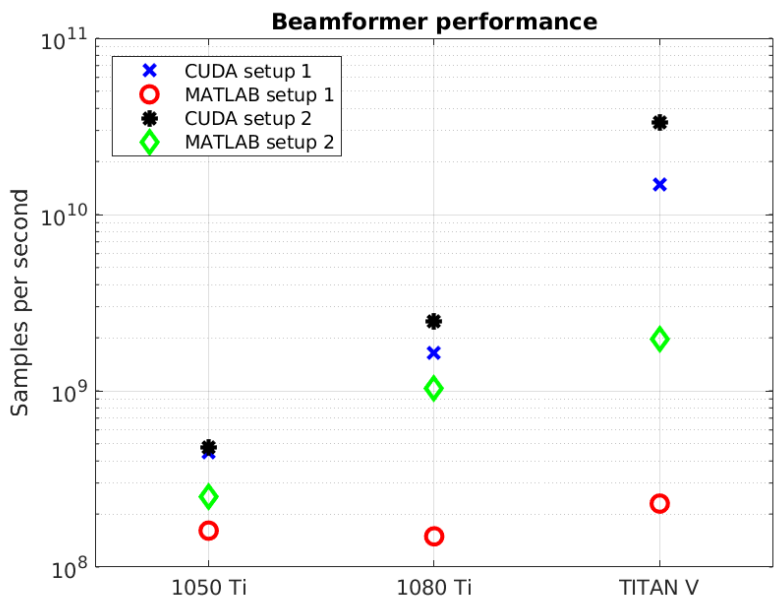

Fig. 2. Performance of the beamformers measured as the number of beamformed samples per second

a computer executing the same sequence of instructions with the same input parameters and data. The non-zero standard deviations are likely the result of using the wall clock time as well as operating system tasks taking up small amounts of processor time.

The CUDA beamformer performs consistently better than the MATLAB beamformer. This is likely due to the MATLAB beamformer being limited by reading and writing intermediate results from and to the GPU's main memory. Each operation (delay and apodization calculations and their constituent operations) translates to a call of a GPU function that reads its operands and writes its result to the GPU's main memory. The main limit for the MATLAB beamformer is, thus, these memory transfers. For the CUDA beamformer, these intermediate results remain in the GPU's internal registers. Another indication of the MATLAB beamformer not being limited by computations is the lack of scaling for setup 1: the TITAN V only performs 1.5 times better than the $1050 \mathrm{Ti}$, whereas it has 33 and 70 times higher performance for the CUDA beamformer with setups 1 and 2, respectively.

Setup 2 has consistently higher sample throughput than setup 1 , even though the two setups have the same input data sets and only differ in the number of output samples. Setup 2 produces around 2.8 million samples, while setup 1 produces around 30 thousand samples. Since the input data is the same in both setups, the time for input data transfer is identical, but many more beamforming operations are performed per data transfer in setup 2. This shows that the data transfers are a significant factor in the performance of setup 1.

This difference in throughput is larger for the MATLAB beamformer than for the CUDA beamformer on all GPUs. This shows that the MATLAB beamformer is more sensitive to the number of output samples than the CUDA beamformer. This is also explained by the relatively lower overhead of performing more computations for each call.

Finally, it is noticed that the worst-case throughput of the CUDA beamformer is still higher than the best-case of the MATLAB beamformer on all GPUs.

\section{DISCUSSION}

For the simulated RC array, full volumes are imaged using only 192 channels. This is in stark contrast to the 36,864 channels required for an equivalent fully populated matrix array.

Linear and curvilinear arrays in clinical use today typically use 192 channels. These arrays, however, only image slices while averaging over the slice thickness that typically varies from $5 \lambda$ at the focus of the fixed elevation lens to 15 to $25 \lambda$ away from the lens depending on the element size and imaging depth. The RC arrays can be focused electronically in both planes yielding an order of magnitude improvement in resolution with the same amount of input data as handled in clinical systems today. This means that interconnect and data transfer do not pose a challenge for 3D imaging using RC arrays. For the examples shown here, each image is comprised of 192 emissions. For setup 1 with the CUDA beamformer on the TITAN V, this means that real-time imaging can be made at a pulse repetition frequency of $2.6 \mathrm{kHz}$.

However, when using $\lambda / 2$-pitch arrays, fewer emissions are needed than for $\lambda$-pitch linear and curvilinear arrays in use today [22], [23]. A study of the number of emissions required to attain similar image quality with $\lambda$ and $\lambda / 2$-pitch probes yielded a reduction from 61 to 21 emissions for plane wave imaging [22]. Similarly, a study of the number of emissions 
required in SA imaging with the same $\lambda / 2$-pitch probe showed minimal differences between using 256 and 32 emissions [23]. This translates directly to an increase in frame rate by a factor of 3 to 8 . This corresponds to frame rates of $52 \mathrm{~Hz}$ to $112 \mathrm{~Hz}$ for setup 1, or 1 to 2.5 full volumes per second for setup 2 with the CUDA beamformer on the TITAN V. This, of course, assumes the beamformer throughput is independent of the number of emissions, which is reasonable since the only added overhead is the read-out of the high-resolution image or volume. This is 45 MBytes for setup 2 using double precision complex values. This should be contrasted to the $15.75 \mathrm{~GB} / \mathrm{s}$ maximum bandwidth of 16-lane PCI Express 3 interfaces used by many contemporary GPUs.

Further speed-ups can be attained by using single-precision floating point numbers or fixed point integer calculations. Experiments using single-precision floating point numbers indicate that the frame rate of setup 1 can be improved from $1.5 \mathrm{~Hz}$ to $13 \mathrm{~Hz}$ for the $1080 \mathrm{Ti}$, and from $14 \mathrm{~Hz}$ to $20 \mathrm{~Hz}$ for the TITAN V. The $1080 \mathrm{Ti}$ has 32 times as high performance for single precision compared to double precision operations due to differences in hardware support for the two [24]. The TITAN V has the same theoretical performance for both precisions. The frame rate increase for the TITAN $\mathrm{V}$ is likely caused by the halved memory bandwidth needed in the beamforming process, while the larger improvement for the $1080 \mathrm{Ti}$ is explained by the improved hardware throughput.

\section{CONCLUSION}

Two row-column beamformers written in the MATLAB programming language and CUDA have been compared. The worst-case performance of the CUDA beamformer exceeds the best-case of the MATLAB beamformer across all GPUs. 3D synthetic aperture imaging is attained at $14 \mathrm{~Hz}$ for two crossplanes with a high-end scientific GPU (TITAN V) for a 192 emission sequence with a $192+192$ array. This corresponds to a pulse-repetition frequency of $2.6 \mathrm{kHz}$ rapidly approaching real-time 3D synthetic aperture imaging. Volumetric synthetic aperture imaging of a $50 \times 50 \times 20 \mathrm{~mm}^{3}$ box at one highresolution volume every three seconds is attained. This volume easily covers e.g. the mitral valve that can be imaged throughout a single heart beat with no need for ECG gating or similar techniques in less than one minute of processing time with only 192 channels of data.

\section{REFERENCES}

[1] S. W. Smith, H. G. Pavy, and O. T. von Ramm, "High speed ultrasound volumetric imaging system - Part I: Transducer design and beam steering," IEEE Trans. Ultrason., Ferroelec., Freq. Contr., vol. 38, pp. 100-108, 1991.

[2] C. E. Morton and G. R. Lockwood, "Theoretical assessment of a crossed electrode 2-D array for 3-D imaging," in Proc. IEEE Ultrason. Symp., 2003, pp. 968-971.

[3] M. F. Rasmussen and J. A. Jensen, "3D ultrasound imaging performance of a row-column addressed 2D array transducer: a simulation study," in Proc. SPIE Med. Imag., 2013, pp. 1-11, 86750C.

[4] H. Bouzari, M. Engholm, C. Beers, M. B. Stuart, S. I. Nikolov, E. V. Thomsen, and J. A. Jensen, "Curvilinear 3-D imaging using row-columnaddressed 2-D arrays with a diverging lens: Feasibility study," IEEE Trans. Ultrason., Ferroelec., Freq. Contr., vol. 64, no. 6, pp. 978-988, 2017.
[5] H. Bouzari, M. Engholm, C. Beers, S. I. Nikolov, M. B. Stuart, E. V. Thomsen, and J. A. Jensen, "Curvilinear 3-D imaging using row-column addressed 2-D arrays with a diverging lens: Phantom study," IEEE Trans. Ultrason., Ferroelec., Freq. Contr., vol. 65, no. 7, pp. 1182-1192, 2018.

[6] J. T. Yen, "Beamforming of sound from two-dimensional arrays using spatial matched filters," J. Acoust. Soc. Am., vol. 134, no. 5, pp. 36973704, 2013.

[7] A. Sampaleanu, P. Zhang, A. Kshirsagar, W. Moussa, and R. Zemp, "Top-orthogonal-to-bottom-electrode (TOBE) CMUT arrays for 3-D ultrasound imaging." IEEE Trans. Ultrason., Ferroelec., Freq. Contr., vol. 61, no. 2, pp. 266-276, 2014.

[8] T. L. Christiansen, M. F. Rasmussen, J. P. Bagge, L. N. Moesner, J. A. Jensen, and E. V. Thomsen, "3-D imaging using row-column-addressed arrays with integrated apodization - part II: Transducer fabrication and experimental results," IEEE Trans. Ultrason., Ferroelec., Freq. Contr. vol. 62, no. 5, pp. 959-971, 2015.

[9] H. Bouzari, M. Engholm, T. L. Christiansen, C. Beers, A. Lei, M. B. Stuart, S. I. Nikolov, E. V. Thomsen, and J. A. Jensen, "Volumetric synthetic aperture imaging with a piezoelectric 2-D row-column probe," in Proc. SPIE Med. Imag., vol. 9790, 2016, pp. 1-9.

[10] S. Holbek, T. L. Christiansen, M. B. Stuart, C. Beers, E. V. Thomsen, and J. A. Jensen, "3-D vector flow estimation with row-column addressed arrays," IEEE Trans. Ultrason., Ferroelec., Freq. Contr., vol. 63, no. 11, pp. 1799-1814, 2016.

[11] S. Holbek, M. B. Stuart, and J. A. Jensen, "Volumetric 3-D vector flow measurements using a 62+62 row-column addressed array," in Proc. IEEE Ultrason. Symp., 2017, pp. 1-4.

[12] W. A. Wulf and S. A. McKee, "Hitting the memory wall: Implications of the obvious," SIGARCH Comput. Archit. News, vol. 23, no. 1, pp. 20-24, mar 1995.

[13] H. K. H. So, J. Chen, B. Y. S. Yiu, and A. C. H. Yu, "Medical ultrasound imaging: to GPU or not to GPU?" IEEE Micro, vol. 31, no. 5, pp. 54-65, 2011.

[14] E. Boni, A. C. H. Yu, S. Freear, J. A. Jensen, and P. Tortoli, "Ultrasound open platforms for next-generation imaging technique development," IEEE Trans. Ultrason., Ferroelec., Freq. Contr., vol. 65, no. 7, pp. 10781092, 2018.

[15] M. F. Rasmussen, T. L. Christiansen, E. V. Thomsen, and J. A. Jensen, "3-D imaging using row-column-addressed arrays with integrated apodization - Part I: Apodization design and line element beamforming," IEEE Trans. Ultrason., Ferroelec., Freq. Contr., vol. 62, no. 5 , pp. 947-958, 2015.

[16] M. B. Stuart, M. Schou, and J. A. Jensen, "Row-column beamforming with dynamic apodizations on a GPU," in Proc. SPIE Med. Imag., 2019, pp. 1-7, paper number 10955-20.

[17] M. Karaman, P. C. Li, and M. O’Donnell, "Synthetic aperture imaging for small scale systems," IEEE Trans. Ultrason., Ferroelec., Freq. Contr. vol. 42, pp. 429-442, 1995.

[18] J. A. Jensen, S. Nikolov, K. L. Gammelmark, and M. H. Pedersen, "Synthetic aperture ultrasound imaging," Ultrasonics, vol. 44, pp. e5e15, 2006.

[19] J. A. Jensen and N. B. Svendsen, "Calculation of pressure fields from arbitrarily shaped, apodized, and excited ultrasound transducers," IEEE Trans. Ultrason., Ferroelec., Freq. Contr., vol. 39, no. 2, pp. 262-267, 1992.

[20] J. A. Jensen, "Field: A program for simulating ultrasound systems," Med. Biol. Eng. Comp., vol. 10th Nordic-Baltic Conference on Biomedical Imaging, Vol. 4, Supplement 1, Part 1, pp. 351-353, 1996.

[21] K. Ranganathan and W. F. Walker, "Cystic resolution: A performance metric for ultrasound imaging systems," IEEE Trans. Ultrason., Ferroelec., Freq. Contr., vol. 54, no. 4, pp. 782-792, 2007.

[22] J. Jensen, M. B. Stuart, and J. A. Jensen, "Optimized plane wave imaging for fast and high-quality ultrasound imaging," IEEE Trans. Ultrason., Ferroelec., Freq. Contr., vol. 63, no. 11, pp. 1922-1934, 2016.

[23] R. Moshavegh, J. Jensen, C. A. Villagomez-Hoyos, M. B. Stuart, M. C. Hemmsen, and J. A. Jensen, "Optimization of synthetic aperture image quality," in Proc. SPIE Med. Imag., vol. 9790, 2016, pp. 97900Z97 900Z-9.

[24] NVIDIA, "Pascal tuning guide," https://docs.nvidia.com/cuda/pascaltuning-guide/index.html, 2018, v10.0.130. 